\title{
SOME REMARKS ON BOUNDARY BEHAVIOR OF ANALYTIC AND MEROMORPHIC FUNCTIONS
}

\author{
F. BAGEMIHL AND W. SEIDEL*
}

1. This paper is concerned with regular and meromorphic functions in $|z|<1$ and their behavior near $|z|=1$. Among the results obtained are the following. In section 2 we prove the existence of a non-constant meromorphic function that tends to zero at every point of $|z|=1$ along almost all chords of $|z|<1$ terminating in that point. Section 3 deals with the impossibility of extending this result to regular functions. In section 4 it is shown that a regular function can tend to infinity along every member of a set of spirals approaching $|z|=1$ and exhausting $|z|<1$ in a simple manner. Finally, in section 5 we prove that this set of spirals cannot be replaced by an exhaustive set of Jordan arcs terminating in points of $|z|=1$; Theorem 3 of this section can be interpreted as a uniqueness theorem for meromorphic functions.

2. THEOREM 1. There exists a function $f(z)$, regular in $|z|<1$, such that, for every $\theta$ in the interval $0 \leqq \theta<2 \pi, f(z) \rightarrow \infty$ as $z \rightarrow e^{i \theta}$ along almost all chords of $|z|<1$ terminating in $e^{i \theta}$.

Proof. Let

$$
n_{j}=3^{j} \quad(j=1,2,3, \ldots),
$$

and define $f(z)$ to be the infinite product ${ }^{2)}$

$$
f(z)=\prod_{j=1}^{\infty}\left\{1-\left(\frac{z}{1-n_{j}^{-1}}\right)^{n_{j}^{2}}\right\}
$$

Received June 17, 1955.

* W. Seidel's contribution to this paper was made under the sponsorship of the National Science Foundation.

1) By this we mean, more pr ecisely, that if $\varphi$ denotes the angle which a chord terminating in $e^{i \theta}$ makes with the radius at $e^{i \theta}$, where $-\pi / 2<\phi<\pi / 2$ and we observe the usual convention regarding the sign of $\varphi$, then the set of values of $\phi$ for which $f(z) \rightarrow \infty$ along the corresponding chord is of Lebesgue measure $\pi$.

2) The use of a product of this form was suggested to us by a product which P. Erdös proposed for another purpose. 
for $|z|<1$. This product converges absolutely and uniformly in every disk $|z| \leqq r<1$, and hence $f(z)$ is regular in $|z|<1$.

The zeros of $f(z)$ are

$$
z_{j, \nu}=\left(1-\frac{1}{n_{j}}\right) e^{2 \pi i \nu / n_{j}^{2}} \quad\left(j=1,2,3, \ldots ; \nu=0,1, \ldots, n_{j}^{\prime}-1\right) .
$$

Let

$$
\rho_{j}=1 / n_{j}^{4} \quad(j=1,2,3, \ldots)
$$

and

$$
\Gamma_{j, \nu}=\left\{z:\left|z-z_{j, \nu}\right|<\rho_{j}\right\} \quad\left(j=1,2,3, \ldots ; \nu=0,1, \ldots, n_{j}^{2}-1\right) .
$$

Denote by $\Delta$ the set obtained by deleting all the disks (4) from $|z|<1$. We shall show that $f(z) \rightarrow \infty$ as $|z| \rightarrow 1$ provided that $z$ remains in $\Delta$.

For an arbitrary integer $k \geqslant 2$, consider the ring

$$
R_{k}=\left\{z: 1-n_{k}^{-1} \leqq|z|<1-n_{k+1}^{-1}\right\},
$$

and decompose the product in (2) into four products $P_{\mu}(\mu=1,2,3,4)$ composed, respectively, of the factors in (2) corresponding to $1 \leqq j \leqq k-1, j=k$, $j=k+1, j \geqslant k+2$, so that

$$
f(z)=\prod_{\mu=1}^{4} P_{\mu}(z)
$$

Let $z_{0} \in R_{k} \cap \Delta$; we are going to determine lower bounds for $\left|P_{\mu}\left(z_{0}\right)\right|(\mu=1$, $2,3,4)$.

First of all, we have

$$
\left|P_{1}\left(z_{0}\right)\right| \geqq \prod_{j=1}^{k-1}\left\{\left(\frac{1-n_{k}^{-1}}{1-n_{j}^{-1}}\right)^{n_{j}^{2}}-1\right\}>e^{n_{k-1} / 3}-1,
$$

where the second inequality is a consequence of the fact that the product is not less than its last factor.

The function $\left|P_{2}(z)\right|$ in the set obtained by deleting the disks $\Gamma_{k, \nu}(\nu=0$, $\left.1, \ldots, n_{k}^{2}-1\right)$ from the plane, attains its minimum at some point $\zeta$ on the frontier of one of these disks. Hence,

$$
\left|P_{2}\left(z_{0}\right)\right| \gtreqless\left|1-\left(\frac{\zeta}{1-n_{k}^{-1}}\right)^{n_{k}^{2}}\right|,
$$

where $\zeta$ is of the form 


$$
\zeta=\left(1-\frac{1}{n_{k}}\right) e^{2 \pi i \nu / n_{k}^{2}}+\rho_{k} e^{i \alpha}
$$

and noting (3), it follows readily that

$$
\left|P_{2}\left(z_{0}\right)\right| \geq c_{1} / n_{k}^{?},
$$

where $c_{1}$, and $c_{2}, \ldots, c_{5}$ in the sequel, denote positive universal constants.

A similar argument shows that

$$
\left|P_{3}\left(z_{0}\right)\right| \geqslant c_{2} / n_{k+1}^{\prime}
$$

Finally, if we set

$$
\prod_{j=1}^{\infty}\left(1-e^{-n_{j}^{2}}\right)=c_{3},
$$

we obtain

$$
\left|P_{4}\left(z_{0}\right)\right| \geqq \prod_{j=k+2}^{\infty}\left\{1-\left(\frac{1-n_{k+1}^{-1}}{1-n_{j}^{-1}}\right)^{n_{j}^{2}}\right\}>\prod_{j=k+2}^{\infty}\left(1-e^{-n_{j}}\right)>c_{3 .} .
$$

The relations (5) to (9) yield

$$
\left|f\left(z_{0}\right)\right|>c_{4} \frac{e^{n_{k-1} / 3}-1}{n_{k}^{2} n_{k+1}^{2}}
$$

and this, in conjunction with (1), shows that as $|z| \rightarrow 1$ with $z \in \Delta, f(z) \rightarrow \infty$.

Now, let $e^{i \theta}$ be any point of $|z|=1$, and fix $k$. The angle subtended at $e^{i \theta}$ by any one of the disks $\Gamma_{k, \nu}\left(\nu=0,1, \ldots, n_{k}^{2}-1\right)$ is at most

$$
2 \operatorname{arc} \sin \left(n_{k} \rho_{k}\right)=2 \operatorname{arc} \sin \left(1 / n_{k}^{3}\right)<c_{5} / n_{k}^{3} .
$$

Since there are $n_{k}^{2}$ of these disks, the sum of the angles subtended at $e^{i \theta}$ by all these disks is less than $c_{5} / n_{k}$.

In view of (1), $\sum_{k=1}^{\infty} n_{k}^{-1}$ is a convergent series. Hence, the set of chords terminating in $e^{i \theta}$ and intersecting infinitely many of the disks $\Gamma_{k, v}(k=1,2$, $\left.3, \ldots ; \nu=0,1, \ldots, n_{k}-1\right)$ is of measure 0 , which means that almost every chord terminating in $e^{i \theta}$ is such that all its points lying in a sufficiently small neighborhood of $e^{i \theta}$ belong to $A$, and this completes the proof of Theorem $1 .^{3)}$

Setting $g(z)=1 / f(z)$, we obtain

3) We remark that $f(\boldsymbol{z})$ also possesses the properties described in Theorems 1 to 4 of F. Bagemihl, P. Erdös, and W. Seidel: Sur quelques propriétés frontières des fonctions holomorphes définies par certains produits dans le cercle-unité, Ann. Sci. École Norm. Sup. (3) 70 (1953), pp. 135-147. 
Corollary 1.) There exists a function $g(z)$, meromorphic and not identically constant in $|z|<1$, such that, for every $\theta$ in the interval $0 \leqq \theta<2 \pi$, $g(z) \rightarrow 0$ as $z \rightarrow e^{i 0}$ along almost all chords of $|z|<1$ terminating in $e^{i \theta}$.

3. Corollary 1 does not remain valid if we replace the word "meromorphic" therein by the word "regular." For suppose that Corollary 1 thus altered were valid. Now Meier ${ }^{51}$ has proved the following theorem:

Let $F(z)$ be regular in $|z|<1$, and $M$ be an arbitrary subset of $|z|=1$. If to every $e^{i \theta} \in M$ there correspond two chords terminating in $e^{i \theta}$ on which $F(z)$ is bounded, then, at almost all points of $M, F(z)$ possesses a finite angular limit.

Meier's theorem applied to $g(z)$ would imply the existence of the angular limit 0 at almost all points of $|z|=1$, which, according to Priwaloff's uniqueness theorem, ${ }^{6)}$ would mean that $g(z) \equiv 0$, contrary to hypothesis.

The function $g(z)$ defined above, just before Corollary 1, shows that Meier's theorem does not remain valid if the word "regular" therein is replaced by the word "meromorphic."

4. Theorem 2. Let $f(z)$ be the regular function in $|z|<1$ defined by (2). Then there exists a set of spirals $S_{t}(0 \leqq t<1)$ in $|z|<1$, each of which approaches $|z|=1$ asymptotically, such that every point of $|z|<1$ belongs to one, and only one, of these spirals, and, for every $t$,

$$
\lim _{\substack{|z| \rightarrow 1 \\ z \in S_{t}}} f(z)=\infty .
$$

Proof. Each spiral $S_{t}(0 \leqq t<1)$ will be defined as the union of enumerably many circular arcs and enumerably many rectilinear segments in $|z|<1$.

For every $r$ with $0<r<1$, let $C_{r}=\{z:|z|=r\}$. It is evident from the simple structure of $\Delta$ (defined above in section 2) that there exists a set of Jordan $\operatorname{arcs} A_{s}(0 \leqq s<1)$ in $\Delta$, each of which is composed of enumerably many

4) This result was announced in F. Bagemihl and W. Seidel: A general principle involving Baire category, with applications to function theory and other fields, Proc. Nat. Acad. Sci. U.S.A. 39 (1953), pp. 1068-1075 (see pp. 1073-1074).

5) K. Meier: Über die Randwerte meromorpher Funktionen und hinreichende Bedingungen für Regularität von Funktionen einer komplexen Variablen, Comm. Math. Helv. 24 (1950), pp. 238-259 (see p. 241).

6) Cf. N. Lusin and J. Priwaloff: Sur l'unicité et la multiplicité des fonctions analytiques, Ann. Sci. École Norm. Sup. (3) 42 (1925), pp. 143-191 (see p. 164). 
rectilinear segments and extends from the point 0 to the point 1 in a strictly monotonic fashion with $|z|$, such that

(i) if $0 \leqq s_{1}<s_{2}<1$, then $A_{s_{1}} \cap A_{s_{2}}=\{0\}$,

(ii) each $A_{s}(0 \leqq s<1)$ lies in the sector of $|z|<1$ defined by $0<\arg z$ $<2 \pi / n_{1}^{2}$ (cf. (1)), and

(iii) if $0<r<1$, then $\left(\bigcup_{0 \leqq s<1} A_{s}\right) \cap C_{r}$ is a subarc of $C_{r}$ described in the clockwise sense by the point $A_{s} \cap C_{r}$ as $s$ varies from 0 to 1 .

Let $\varphi_{k}(t)(k=1,2,3, \ldots)$ be a one-to-one order-reversing mapping of $0 \leqq t<1$ onto $(k-1) / k<s \leqq k /(k+1)$. Denote by $\psi_{1}(t)$ a one-to-one orderreversing mapping of $0 \leqq t<1$ onto $0<r \leqq 1-n_{2}^{-1}-n_{2}^{-4}$ such that $\psi_{1}(1 / 2)$ $=1-n_{1}^{-1}+n_{1}^{-4}$; and by $\varphi_{k}(t)(k=2,3,4, \ldots)$ a one-to-one order-reversing mapping of $0 \leqq t<1$ onto $1-n_{k}^{-1}-n_{k}^{-4}<r \leqq 1-n_{k+1}^{-1}-n_{k+1}^{-4}$ such that $\psi_{k}(k /(k+1))=1-n_{k}^{-1}+n_{k}^{-4}$.

Define $E_{t}^{(1)}(0 \leqq t<1)$ to be the subarc of $A_{p_{1}(t)}$ extending from the origin (exclusive) to $C_{\psi_{1}(t)}$ (inclusive), and $E_{t}^{(k)}(k=2,3,4, \ldots ; 0 \leqq t<1)$ to be the subarc of $A_{p_{k}(t)}$ extending from $C_{\psi_{k-1}(t)}$ (exclusive) to $C_{\psi_{i k}(t)}$ (inclusive). Take $F_{t}^{(k)}(k=1,2,3, \ldots ; 0 \leqq t<1)$ to be the subarc of $C_{\psi_{k}(t)}$ extending in the counterclockwise sense from $A_{\varphi_{k}(t)}$ (exclusive) to $A_{f_{k+1}(t)}$ (inclusive).

Now set

$$
S_{0}=\{0\} \cup\left(\bigcup_{k=1}^{\infty}\left(E_{0}{ }^{(k)} \cup F_{0}{ }^{(k)}\right)\right)
$$

and

$$
S_{t}=\bigcup_{k=1}^{\infty}\left(E_{t}^{(k)} \cup F_{t}^{(k)}\right) \quad(0<t<1)
$$

For every $k=1,2,3, \ldots$, if $(k-1) / k \leqq t<k /(k+1)$, then for all $z \in S_{t}$ with $|z|>1-n_{k}^{-1}+n_{k}^{-4}$ we have $z \in \Delta$, so that (11) holds, and it is evident from the foregoing construction that the remaining conditions in Theorem 2 are also satisfied.

Setting $g(z)=1 / f(z)$ again, we obtain

Corollary 2. There exists a function $g(z)$, meromorphic and not identically constant in $|z|<1$, and a set of spirals $S_{t}(0 \leqq t<1)$ in $|z|<1$, each of which approaches $|z|=1$ asymptotically, such that every point of $|z|<1$ belongs to one, and only one, of these spirals, and, for every $t$, 


$$
\lim _{\substack{|z| \rightarrow 1 \\ z \in s_{t}}} g(z)=0
$$

5. Theorem 2 and Corollary 2 are no longer valid if the set of spirals $S_{t}$ $(0 \leqq t<1)$ is replaced by an analogous set of Jordan arcs in $|z|<1$ terminating in the points of $|z|=1$. This is an immediate consequence of our next theorem.

Let $Q$ be a point of $|z|<1$, and consider a set of Jordan $\operatorname{arcs} J_{\theta}\left(0 \leqq \theta^{\prime}\right.$ $<2 \pi)$ in $|z|<1, J_{\theta}$ extending from $Q$ to $e^{\imath \theta}$, such that every point of $|z|<1$ belongs to one, and only one, of these arcs. ${ }^{7)}$ Denote the Riemann sphere by $Z$. If $h(z)$ is a function defined in $|z|<1$, the cluster set of $h(z)$ at a point $e^{i \theta}$ of $|z|=1$ is defined to be the set of all points $w \in Z$ with the property that there exists a sequence of points $\left\{z_{n}\right\}$ in $|z|<1$ for which $z_{n} \rightarrow e^{i \theta}$ and $h\left(z_{n}\right) \rightarrow w$ as $n \rightarrow \infty$; and the cluster set of $h(z)$ on $J_{\theta}$ at $e^{i \theta}$ is defined to be the set of all points $w \in Z$ with the property that there exists a sequence of points $\left\{z_{n}\right\}$ on $J_{\theta}$ for which $z_{n} \rightarrow e^{i \theta}$ and $h\left(z_{n}\right) \rightarrow w$ as $n \rightarrow \infty$.

We first note

Lemma 1. Let $h(z)$ be continuous ${ }^{8)}$ in $|z|<1$, and $A$ be a subarc of $|z|=1$. If the cluster set of $h(z)$ at every point of $A$ is $Z$, then there exists a residual ${ }^{9)}$ subset, $R$, of $A$ such that, for every $e^{i \theta} \in R$, the cluster set of $h(z)$ on $J_{\theta}$ at $e^{i \theta \text {. }}$ is $Z$.

Proof. This lemma is an immediate consequence of Theorem 2 in the paper of the present authors' referred to in footnote ${ }^{4)}$, if, in that theorem, we identify $\mathscr{H}_{1}$ with $\{z:|z|<1\}, \mathscr{H}_{2}$ with $Z, \mathcal{S}$ with $A, H_{s}$, for every $s=e^{i \theta} \in \mathcal{S}$, with $J_{\theta}$, and $f$ with $h(z)^{10)}$

THEOREM 3. Let $H$ be a nonempty subset of $Z$ of harmonic measure ${ }^{11)}$ zero, $A$ be a subarc of $|z|=1$, and $h(z)$ be meromorphic and not identically constant

7) We regard $Q$ as belonging to $J_{0}$ but to no other $J_{\theta}(0<\theta<2 \pi)$.

8) By continuous we mean continuous in the extended sense, so that, e.g., a meromorphic function is to be regarded as continuous at a pole.

9) A subset of $A$ is of first category if it is the union of enumerably many nowhere dense subsets of $A$, and a residual subset of $A$ is the complement (with respect to $A$ ) of a subset of $A$ of first category.

10) We take this opportunity to note that, in the aforementioned paper of the present authors', Theorems 5, 6, and 9, which are stated there for meromorphic functions, are obviously valid if the functions are assumed to be merely continuous.

11) For the notion of harmonic measure, see, e.g., R. Nevanlinna: Eindeutige analytische Funktionen, 2nd ed., Berlin-Göttingen-Heidelberg, 1953. 
in $|z|<1$. Suppose that the subset $P$ of $A$ is of positive measure on every subarc of $A$, and that, for every $e^{i \theta} \in P$,

$$
\lim _{\substack{z \rightarrow \boldsymbol{i n}^{i \theta} \\ z \in J_{\theta}}} h(z)=\lambda(\theta)
$$

exists and belongs to $H$. Then there exists a residual subset, $R$, of $A$ such that, for every $e^{i \theta} \in R$, the cluster set of $h(z)$ on $J_{0}$ at $e^{i \theta}$ is $Z$.

Proof. According to Lemma 1, it is sufficient to show that the cluster set of $h(z)$ at every point of $A$ is $Z$. Suppose that, on the contrary, the cluster set of $h(z)$ at some point $z_{0} \in A$ is not $Z$. Then, since this cluster set is closed, there exists a neighborhood, $U_{b}$, of a finite point $b$, and a neighborhood, $U_{z_{b}}$, of $z_{0}$, such that, in $V_{z_{0}}=U_{z_{0}} \cap\{z:|z|<1\}, h(z)$ omits every value in $U_{b}$. Since $1 /(h(z)-b)$ is bounded in $V_{z_{0}}, h(z)$, by an obvious extension of Fatou's theorem, possesses limits (finite or infinite) along almost all radii of $|z|<1$ terminating in points of $P \cap V_{z_{0}}$. All but an at most enumerable number of these limits must belong to $H^{12)}$ so that $h(z)$, by the Frostman-Nevanlinna theorem, ${ }^{13)}$ is identically constant, contrary to hypothesis.

The Institute for Advanced Study

University of Notre Dame

12) F. Bagemihl: Curvilinear cluster sets of arbitrary functions, Proc. Nat. Acad. Sci. U.S.A. 41 (1955), pp. 379-382, Theorem 2.

13) Cf. R. Nevanlinna: loc. cit. ${ }^{11)}$, p. 209. 